\title{
Wege zur besseren Eigenkapitalausstattung
}

\author{
Stefan Kusterer
}

Trotz des Gebots der Selbstlosigkeit sind auch gemeinnützige Organisationen für ihre wirtschaftliche Entwicklung auf genügend Eigenkapital angewiesen. Ein schwieriger aber gangbarer Weg ist die Ergänzung durch »Mezzaninkapital «.

Gemeinnützige Organisationen sind in den unterschiedlichsten Rechtsformen organisiert. Neben dem gemeinnützigen eingetragenen Verein und der gemeinnützigen Stiftung sind zunehmend auch gemeinnützige $\mathrm{GmbHs}$ und gemeinnützige Aktiengesellschaften vorzufinden. Allen gemeinnützigen Organisationen gemein ist das in $§ 55$ der Abgabenordnung (AO) verankerte Gebot der Selbstlosigkeit, das im Kern besagt, dass eine gemeinnützige Organisation nicht überwiegend erwerbswirtschaftlich arbeiten darf. Nicht zuletzt dieser Umstand führt dazu, dass gemeinnützige Organisationen in der Regel über eine geringe Eigenkapitaldecke verfügen.

In Wirklichkeit sind gemeinnützige Organisationen zur Sicherung ihres Fortbestands jedoch wie Wirtschaftsunternehmen auf Wachstumsfinanzierung und somit auf Kapitalgeber angewiesen. Zusätzliches Eigenkapital kann praktisch ohnehin nur dann beschafft werden, wenn die Organisation, bei der sich weitere Eigenkapitalgeber engagieren sollen, schon in der Ausgangslage über eine attraktive Eigenkapitalquote verfügen. Denn Investoren erscheint es regelmäßig nur dann attraktiv, Eigenkapital zur Verfügung zu stellen, wenn andere bereits als Eigenkapitalgeber engagiert sind.

Somit ist zum einen zu klären, wie die Eigenkapitalquote gemeinnütziger Organisationen in der Ausgangslage verbessert werden kann. Zum anderen stellt sich die Frage, wie zusätzliches Eigenkapital beschafft werden kann und welche Formen hierfür zur Verfügung stehen.

\section{Verbesserung der Eigenkapitalquote durch Hebung stiller Reserven}

Grundsätzlich kann die Eigenkapitalquote durch die Realisierung stiller Reserven verbessert werden, weil die Differenz zwischen dem Zeitwert und dem niedrigeren Buchwert eines Vermögensteils ohne dessen Verkauf erfolgswirksam zugunsten des Eigenkapitals aufgedeckt wird. Hierbei handelt es sich also um eine handelsbilanzielle Maßnahme, mit der kein Liquiditätszufluss von außen verbunden ist. Für Dritte, die sich mit möglichen Investitionen in gemeinnützige Organisationen befassen,

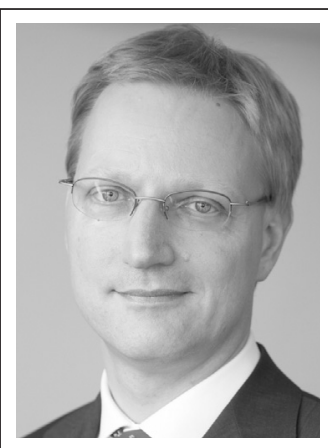

Dr. Stefan Kusterer ist Wirtschaftsprüfer und Steuerberater und seit 2001 geschäftsführender Gesellschafter der Susat \& Partner OHG mit Dienstsitz in München. Nach dem Studium der Betriebswirtschaftslehre und der Promotion auf dem Gebiet des Konzernrechts liegen seine Beratungsschwerpunkte heute auf den Gebieten des Umwandlungs- und Umwandlungssteuerrechts, des Gemeinnütigkeitrechts sowie der Unternehmensnachfolge. Darüber hinaus ist er Verfasser einer Vielzahl von Beiträgen in Fachzeitschriften und Mitverfasser eines aktienrechtlichen Handbuchs und eines handelsrechtlichen Kommentars.

E-Mail S.Kusterer@susat.de

ist dieser Sachverhalt gleichwohl von Gewicht, weil das Verhältnis von Eigen- zu Fremdkapital in der Handelsbilanz dokumentiert ist und die Organisation nicht von Haus aus den Anschein der Abhängigkeit von Fremdkapitalgebern vermittelt.

Im ideellen Bereich einer gemeinnützigen Organisation werden stille Reserven kaum vorzufinden sein. Dagegen können im Bereich der Vermögensverwaltung und im Bereich des wirtschaftlichen Geschäftsbetriebs oder des Zweckbetriebs erhebliche Reserven ruhen, etwa weil die Organisation über seit dem Erwerb wertvoll gewordene Beteiligungen oder Grundbesitz verfügt.

Unter steuerlichen Gesichtspunkten ist zu berücksichtigen, dass der wirtschaftliche Geschäftsbetrieb keinen Steuerbegünstigungen unterliegt. Die Hebung stiller Reserven in diesem Bereich würde deshalb Ertragsteuern auslösen und kommt deshalb praktisch nicht in Betracht. Die Vermögensverwaltung und die Zweckbetriebe stellen dagegen - neben dem hier nicht in Betracht kommenden ideellen Bereich — die steuerbegünstigten Sphären einer gemeinnützigen Organisation dar, wobei sich die Steuerbegünstigungen insbesondere auf die Körperschaftsteuer ( 55 Abs. 1 Nr. 9 KStG) und die Gewerbesteuer ( $\$ 3$ Nr. 6 GewStG) beziehen, daneben aber auch die Grundsteuer, die Erbschaft- und Schenkungsteuer und den Umsatzsteuerbereich betreffen. Mit der Aufdeckung stiller Reserven in den Sphären der Vermögensverwaltung 
und des Zweckbetriebs ist dementsprechend kein Liquiditätsabfluss für Ertragsteuern verbunden.

Zweckmäßigerweise werden die stillen Reserven in vermögensverwaltendem Vermögen und in Zweckvermögen realisiert, indem die gemeinnützige Organisation dieses Vermögens zu den über den Buchwerten liegenden Zeitwerten in eine Tochtergesellschaft einbringt. Der — steuerfreie - Einbringungsgewinn wird dann bei der übertragenden Organisation erfolgswirksam vereinnahmt und erhöht so, im Ergebnis etwa durch die Bildung von Gewinnrücklagen, das Eigenkapital.

Bei der Wahl der Rechtsform des Rechtsträgers, der das Vermögen übernehmen soll (»Übernehmer«), ist allerdings Sorgfalt geboten. Unter steuerlichen Gesichtspunkten kann das Ziel der Transaktion nicht darin bestehen, dass das aus einem steuerbegünstigten Bereich übertragene Vermögen auf einen Rechtsträger übergeht, dessen Rechtsform oder Ausgestaltung dazu führt, dass die an ihm gehaltene Beteiligung dem wirtschaftlichen Geschäftsbetrieb der gemeinnützigen Organisation zuzuordnen ist. Die Realisierung stiller Reserven darf also nicht dadurch erkauft werden, dass ursprünglich steuerbegünstigtes Vermögen in nicht steuerbegünstigtes Vermögen umgewidmet wird. Deshalb kommt als Übernehmer weder eine Kapitalgesellschaft in Betracht, an der der Übertrager mehrheitlich beteiligt ist, noch eine gewerblich tätige oder gewerblich geprägte Personengesellschaft.

In der Praxis bietet sich, wenn auch nicht ganz unumstritten, aber gegenüber der Finanzverwaltung durchsetzbar, als Rechtsform des Übernehmers somit eine Kapitalgesellschaft \& Co. KG an, bei der der Kommanditist geschäftsführungsbefugt ist. Nach $\S 15$ Abs. 3 Nr. 2 EStG handelt es sich bei einer derart ausgestalteten Kapitalgesellschaft \& Co. KG um eine nicht gewerblich geprägte Personengesellschaft.

Die Kommanditbeteiligung an der Kapitalgesellschaft \& Co. KG, die von der gemeinnützigen Organisation gehalten und durch die Einbringung von Vermögen mit Leben erfüllt wird, ist bei der gemeinnützigen Organisation der Vermögensverwaltung oder dem Zweckbetrieb zuzuordnen. Steuerbegünstigtes Vermögen der gemeinnützigen Organisation wird durch die Überführung auf eine Tochtergesellschaft also in eine steuerbegünstigte Beteiligung transformiert. Dabei ist erheblich, dass die Beteiligungsgesellschaft hierfür keineswegs selbst gemeinnützig sein muss. Eine vermögensverwaltende Tätigkeit der Beteiligungsgesellschaft genügt.

\section{Beschaffung von zusätzlichem Eigenkapital}

Zusätzliches Eigenkapital für gemeinnützige Organisationen kann grundsätzlich - beispielhaft eine gemeinnützige GmbH oder AG unterstellt - wie bei Wirtschaftsunternehmen in zusätzlichem Stamm- bzw. Grundkapital bestehen. Allerdings können gemeinnützige Organisationen wegen des Grundsatzes der Selbstlosigkeit weder Gewinnausschüttungen vornehmen noch einen etwaigen Liquidationserlös an die Eigenkapitalgeber auskehren, wenn es sich bei den Eigenkapitalgebern nicht selbst um gemeinnützige Organisationen handelt. Der ökonomische
Wert eines Geschäftsanteils oder einer Aktie an einer gemeinnützigen GmbH bzw. AG ist für einen nicht gemeinnützigen Investor deshalb null. Darin liegt zugleich der Grund, warum Investoren in aller Regel nicht dafür gewonnen werden können, Stammeinlagen oder Aktien einer gemeinnützigen Organisation zu zeichnen.

Als zusätzliches Eigenkapital kommt deshalb jenes Kapital in Betracht, das unter dem Begriff »Mezzaninkapital« diskutiert wird. Mezzaninkapital führt zu einem Liquiditätszufluss, der auf einer schuldrechtlichen Grundlage beruht, die so ausgestaltet ist, dass die zugeführte Liquidität in der Handelsbilanz des Kapitalnehmers als Eigenkapital ausgewiesen werden kann. Die hierfür im einzelnen zu erfüllenden Voraussetzungen sind in der Stellungnahme des Hauptfachausschusses des Instituts der Wirtschaftsprüfer in Deutschland 1/1994 (HFA/IDW 1/1994) zusammengefasst und stellen insbesondere auf die Langfristigkeit der Kapitalüberlassung, die Nachrangigkeit und die Erfolgsabhängigkeit der Vergütung des überlassenen Kapitals ab. Im Kern wird der Ausweis als Eigenkapital also damit begründet, dass mezzanines Kapital wirtschaftlich wie Eigenkapital behandelt wird, ohne auf gesellschaftsrechtlicher, sondern lediglich schuldrechtlicher Grundlage zu beruhen. Als Formen von mezzaninem Kapital haben sich in der Praxis insbesondere die Genussrechte und die stille Beteiligung herausgebildet, weil für ihre Ausgestaltung hinreichend große Spielräume bestehen.

Fraglich ist dann, ob gemeinnützige Organisationen Mezzaninkapital erwerben können, ohne den Status der Gemeinnützigkeit zu gefährden. Dagegen spricht insbesondere, dass eine Anforderung für den Ausweis von Mezzaninkapital als Eigenkapital in der Erfolgsabhängigkeit der Vergütung besteht. In aller Regel wird der Erfolg an nachvollziehbaren ökonomischen Größen wie dem Jahresergebnis vor der (als Aufwand zu berücksichtigenden) Vergütung des Mezzaninkapitals gemessen, so dass die Vergütung des Mezzaninkapitals mit zunehmendem (modifiziertem) Jahresergebnis steigt. Ein solcher Maßstab ist wegen der Eigenkapitalähnlichkeit von Mezzaninkapital auch sachgerecht. Indessen kann ein solcher oder ähnlicher Erfolgsmaßstab von gemeinnützigen Organisationen ohne Gefährdung der Gemeinnützigkeit kaum vereinbart werden, denn die Maximierung von - wie auch immer gearteten - Jahresergebnissen kann angesichts der Voraussetzungen für Gemeinnützigkeit gerade nicht die Handlungsmaxime gemeinnütziger Organisationen bilden. Die Frage, ob gemeinnützige Organisationen Mezzaninkapital erwerben können, hängt deshalb davon $\mathrm{ab}, \mathrm{ob}$ im Einzelfall gemeinnützigkeitsverträgliche Erfolgsmaßstäbe für die Vergütung von Mezzaninkapital gefunden werden, die den Anforderungen eines Eigenkapitalausweises genügen.

Die oben vorgenommene Analyse der steuerfreien Realisierung stiller Reserven hat jedoch ergeben, dass gemeinnützige Organisationen durchaus Vermögen auf einen anderen Rechtsträger übertragen können, der selbst nicht gemeinnützig ist, wobei die an ihm gehaltene Beteiligung dem steuerbegünstigten Vermögen der gemeinnützigen Organisation zuzurechnen ist. Solche Rechtsträger handeln, auch wenn sie vermögensverwaltend tätig sind, nach dem ökonomischen Prinzip. Sie können deshalb nicht nur Mezzaninkapital, sondern auch Eigenkapital 
auf gesellschaftsrechtlicher Grundlage ausgeben, ohne dass die Gemeinnützigkeit der an diesen Rechtsträgern beteiligten Organisation gefährdet wird.

Entscheidend ist also, dass eine gemeinnützige Organisation auf eine Tochtergesellschaft Vermögen auslagern kann und diese Tochtergesellschaft dann in der Lage ist, zusätzliches Eigenkapital durch Hereinnahme von Investoren zu generieren. Zugleich kann die gemeinnützige Organisation die Vermögensauslagerung auf die Tochtergesellschaft nutzen, um durch Realisierung stiller Reserven ihr eigenes Eigenkapital im Wege der Innenfinanzierung zu erhöhen.

\section{Zusammenfassung und Ausblick}

(1) Gemeinnützige Organisationen sind in der Lage, ihre Eigenkapitalquote durch die steuerfreie Aufdeckung stiller Reserven zu verbessern, indem sie geeignetes Vermögen auf einen anderen sachgerecht ausgestalteten Rechtsträger übertragen, der durch diese Vermögensübertragung in einem Tochterverhältnis zur gemeinnützigen Organisation steht.

(2) Regelmäßig können sich gemeinnützige Organisationen keine zusätzliche Liquidität durch die gesellschaftsrechtliche Erhöhung ihres Eigenkapitals, das z.B. in Form von Stammkapital oder Grundkapital bestehen kann, beschaffen, insbesondere weil das Gemeinnützigkeitsrecht Gewinnausschüttungen an selbst nicht gemeinnützige Investoren ausschließt. Die Hereinnahme von sogenanntem mezzaninem Kapital, das in der Handelsbilanz unter bestimmten Voraussetzungen als Eigenkapital ausgewiesen werden kann, ist für gemeinnützige
Organisationen im Einzelfall für bestimmte Modelle der Vergütung von Mezzaninkapital statthaft.

(3) Der Königsweg wird darin bestehen, dass eine Tochtergesellschaft der gemeinnützigen Organisation für die Hereinnahme von mezzaninem Kapital zu Verfügung gestellt wird. Diese Tochtergesellschaft kann zum einen gemeinnützigkeitsverträglich für die Mutterorganisation ausgestaltet werden, ohne selbst gemeinnützig zu sein, und zum anderen zur Eigenkapitalausstattung durch Innenfinanzierung im Wege der Realisierung stiller Reserven zur Verfügung stehen.

(4) Auch wenn sich die beschriebenen Modelle in der Praxis bewährt haben und vollzogen werden, muss abschließend eindringlich davon abgeraten werden, derartige Gestaltungen ohne Abstimmung mit der Finanzverwaltung zu realisieren. Gestaltungen wie die beschriebenen müssen akribisch durchdacht, vorbereitet und vollzogen werden, um unliebsame Überraschungen von Seiten der Finanzverwaltung zu vermeiden.

\section{Was ist eigentlich »Mezzaninkapital«?}

Mezzaninkapital ist der Sammelbegriff für Finanzierungsarten, bei denen in der klassischen Variante einem Unternehmen Eigenkapital zugeführt, ohne den Kapitalgebern Stimm- oder Einflussnahmerechte zu gewähren. Mezzaninkapital kann beispielsweise aus Genussrechten oder stillen Beteiligungen, aus Wandelund Optionsanleihen bestehen. Mezzaninkapital steht - wie das Zwischengeschoss (»Mezzanin«) eines Hauses - zwischen Eigen- und Fremdkapital.

\title{
Kernprozesse des Wissensmanagements
}

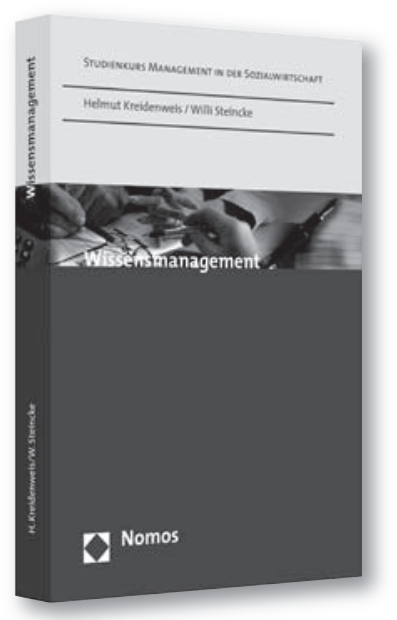

\author{
Wissensmanagement \\ Von Prof. Helmut Kreidenweis, Hochschule Neubrandenburg \\ und Willi Steincke \\ 2005, 161 S., brosch., 19,80 €, ISBN 978-3-8329-1613-8 \\ (Studienkurs Management in der Sozialwirtschaft)
}

Dieses Buch zeigt, welche Arten von Wissen es gibt und was beim Management von Wissen in sozialen Organisationen berücksichtigt werden muss. Die Kernprozesse des Wissensmanagements werden ebenso verständlich dargestellt wie Strategien und Methoden zur Einführung. Der informationstechnologischen Unterstützung widmet sich ein eigenes Kapitel. Ein Workshop-Konzept zum Kennenlernen der Sichtweisen und Methoden des Wissensmanagements ergänzt den Band. 